\title{
Amyloid Precursor-like Protein 2 Association with HLA Class I Molecules
}

\author{
Amit Tuli ${ }^{1,2}$, Mahak Sharma ${ }^{2}$, Xiaojian Wang ${ }^{1}$, Laura C. Simone ${ }^{1}$, Haley L. Capek ${ }^{1}$, Steven \\ Cate $^{3}$, William H. Hildebrand ${ }^{3}$, Naava Naslavsky ${ }^{2}$, Steve Caplan ${ }^{2}$, and Joyce C. Solheim $1,2,{ }^{*}$ \\ ${ }^{1}$ Eppley Institute for Research in Cancer and Allied Diseases, University of Nebraska Medical \\ Center, Omaha, NE, USA \\ ${ }^{2}$ Department of Biochemistry and Molecular Biology, University of Nebraska Medical Center, \\ Omaha, NE, USA \\ ${ }^{3}$ Department of Microbiology and Immunology, University of Oklahoma Health Sciences Center, \\ Oklahoma City, OK, USA
}

\begin{abstract}
Amyloid precursor-like protein 2 (APLP2) is a ubiquitously expressed protein. The previously demonstrated functions for APLP2 include binding to the mouse major histocompatibility complex (MHC) class I molecule $\mathrm{H}-2 \mathrm{~K}^{\mathrm{d}}$ and down regulating its cell surface expression. In this study, we have investigated the interaction of APLP2 with the human leukocyte antigen (HLA) class I molecule in human tumor cell lines. APLP2 was readily detected in pancreatic, breast, and prostate tumor lines, although it was found only in very low amounts in lymphoma cell lines. In a pancreatic tumor cell line, HLA class I was extensively co-localized with APLP2 in vesicular compartments following endocytosis of HLA class I molecules. In pancreatic, breast, and prostate tumor lines, APLP2 was bound to the HLA class I molecule. APLP2 was found to bind to HLA-A24, and more strongly to HLA-A2. Increased expression of APLP2 resulted in reduced surface expression of HLA-A2 and HLA-A24. Overall, these studies demonstrate that APLP2 binds to the HLA class I molecule, colocalizes with it in intracellular vesicles, and reduces the level of HLA class I molecule cell surface expression.
\end{abstract}

\section{Keywords}

amyloid precursor-like protein 2; antigen presentation; human leukocyte antigen; major histocompatibility complex class I; tumor

\section{Introduction}

Amyloid precursor-like protein 2 (APLP2) is a ubiquitous protein anchored at the plasma membrane by a transmembrane domain, which can be cleaved to permit secretion of the APLP2 ectodomain (43). Increased levels of APLP2 have been reportedly expressed by some tumors $(1,10)$. APLP2 was found to be one of the genes most often differentially expressed in a comparison between cases of ductal carcinoma in situ and invasive ductal carcinoma, with an average expression level 13.7-fold higher in invasive ductal carcinoma (1).

*Corresponding Author Email: E-mail: jsolheim@unmc.edu. 
APLP2 is active in several physiological processes, including cell adhesion, migration, cell signaling, and cell cycle regulation $(6,16,20,32,49,55)$. APLP2 is conserved between mouse and human, and also bears homology to two other proteins in mammals (amyloid precursor protein and amyloid precursor-like protein 1) and to proteins expressed by Drosophila melanogaster and Caenorhabditis elegans (55). Amyloid precursor protein influences endocytosis of the high-affinity choline transporter, and APLP2 has also been shown to interact with this transporter, although whether APLP2 is also involved in its endocytosis is unknown (56). Despite the production of several knock-out mouse strains lacking either one or several members of this protein family (2), the full physiological function of APLP2 remains poorly understood.

Previous studies from our laboratory and others have indicated that APLP2 binds to the mouse MHC class I molecule $\mathrm{H} 2-\mathrm{K}^{\mathrm{d}}$ and regulates its expression at the plasma membrane $(13,24$, 39,51,52). Interaction between $H 2-K^{d}$ and APLP2 is dependent on the presence of $\beta_{2^{-}}$ microglobulin (39). APLP 2 binds to the folded $\alpha 1 / \alpha 2$ domain region and to a site in the $\alpha 3 /$ transmembrane/cytoplasmic region of $\mathrm{H} 2-\mathrm{K}^{\mathrm{d}}(51)$. Furthermore, APLP2 modulates the stability and turnover of $\mathrm{H} 2-\mathrm{K}^{\mathrm{d}}$ molecules (52). By transfection systems in human cells, it was shown that elevated expression of APLP2 reduces surface $\mathrm{H} 2-\mathrm{K}^{\mathrm{d}}$ expression, whereas APLP2specific siRNA transfection increased surface expression of $\mathrm{H} 2-\mathrm{K}^{\mathrm{d}}$ and, to a lesser extent, $\mathrm{H} 2-$ $\mathrm{L}^{\mathrm{d}}(24,51,52)$. Interactions between APLP2 and HLA class I molecules have been previously reported, but the interactions were described as weak, the HLA class I allotypes that were tested were not identified, and the data were not shown (39).

Here, we report data showing that APLP2 can also bind to HLA class I molecules in human tumor cells. APLP2 and HLA class I molecules were found co-localized within intracellular vesicular compartments in tumor cells after HLA class I endocytosis. APLP2 co-

immunoprecipitated with HLA class I molecules, and exhibited stronger binding to the HLAA2 allotype than to the HLA-A24 allotype. Finally, elevated intracellular expression of APLP2 resulted in decreased surface expression of HLA class I molecules, suggesting that APLP2 down-regulates HLA class I expression at the plasma membrane. These results suggest a possible role for APLP2 in regulating HLA class I expression on human tumor cells.

\section{Materials and methods}

\section{Antibodies}

HC10 is a monoclonal antibody directed against unfolded human MHC class I heavy chains $(7,38,45,46)$. W6/32 is a conformation-dependent monoclonal antibody that detects folded, $\beta_{2} \mathrm{~m}$-associated HLA class I molecules $(7,24,36)$. Hybridomas producing the W6/32 and HC10 antibodies used for immunoprecipitations and Western blots (respectively) were donated by Dr. Ted Hansen (Washington University, St. Louis, MO, USA). For the immunofluorescence experiments with human cells, a purified form of the W6/32 antibody (anti-human HLA-A,B,C antibody) was obtained from Leinco Technologies (St. Louis, MO, USA). The antibody that was used for immunoprecipitations of HLA-A24 and flow cytometry on 721.221 transfectants was an IgG2b monoclonal antibody from One Lambda, Inc. (Canoga Park, CA, USA) that binds both to HLA-A24 and-A23 subtypes. The antibody used for detection of HLA-A24 on MDA-MB435S cells in flow cytometry experiments was the A11.M antibody (14), which recognizes HLA-24 and HLA-A11; the A11.M hybridoma was purchased from ATCC (Manassas, VA, USA). The BB7.2 antibody (30), which recognizes HLA-A2, was produced from a hybridoma obtained from ATCC (Manassas, VA, USA). The antibody recognizing fulllength APLP2 was purchased from Calbiochem/EMD Chemicals (San Diego, CA, USA). The anti-FLAG rabbit antiserum was obtained from Cell Signaling Technology, Inc. (Beverly, MA, USA). The antibody for the transferrin receptor was obtained from BD Pharmingen. The antibody against EEA1, an early endosome marker (25), was purchased from Transduction 
Laboratories. Secondary antibodies for immunofluorescence were bought from Invitrogen Molecular Probes (Carlsbad, CA, USA).

\section{Cell lines}

The human cell lines used in this study were cultured in RPMI 1640 medium (Invitrogen, Carlsbad, CA, USA) that was supplemented with $15 \%$ (vol/vol) fetal bovine serum, $1 \mathrm{mM}$ sodium pyruvate, $2 \mathrm{mM}$ L-glutamine, $100 \mathrm{units} / \mathrm{ml}$ penicillin, and $100 \mu \mathrm{g} / \mathrm{ml}$ streptomycin. The media of stable transfectants was also supplemented with $0.4 \mathrm{mg} / \mathrm{ml} \mathrm{G} 418$. The HeLa cell line (36) was a gift from Dr. Wendy Maury (University of Iowa, Iowa City, IA, USA). MCF-7 and MDA-MB435S breast tumor cell lines $(3,44)$ were obtained from Dr. Kenneth Cowan and Dr. Vinod Labhasetwar, respectively (University of Nebraska Medical Center, Omaha, NE, USA). The human pancreatic tumor cell lines S2-013, SUIT2, and Hs766T $(18,28,50)$ were donated by Dr. Michael A. Hollingsworth (University of Nebraska Medical Center, Omaha, NE, USA). S2-013 is a cloned subline of the SUIT-2 human pancreatic tumor cell line, which was originally derived from a liver metastasis (18). The Huh7 hepatoma cell line and DU145 prostate tumor cell lines $(26,48)$ were gifts from Dr. Richard MacDonald (University of Nebraska Medical Center, Omaha, NE, USA). The CL-01 and SU-DHL-6 lymphoma cell lines $(9,12)$ were donated by Dr. John Chan (University of Nebraska Medical Center, Omaha, NE, USA). The A673 Ewing's tumor cell line was purchased from ATCC (Manassas, VA, USA) (22). The 721.221 cell line is a mutant B lymphoblastoid cell line reported not to express HLAA, -B, or -C (41,42), and which was a gift from Dr. Ted Hansen (Washington University, St. Louis, MO, USA).

The 721.221 cell line and the HeLa cell line were stably transfected with the HLA-A*2402 cDNA (23) in the pcDNA 3.1(+) vector (Invitrogen, Carlsbad, CA, USA) (a gift from Drs. Atsunori Hiasa and Hiroshi Shiku, Mie University School of Medicine, Mie, Japan) with the use of the Amaxa Nucleofector (Amaxa, Inc., Gaithersburg, MD, USA). Transient transfections of the HeLa-A24 cells with APLP2 in the pCMV-Tag4A vector (Stratagene, Cedar Creek, TX, USA) were performed with Effectene (Qiagen, Inc., Valencia, CA, USA) at 24 hours before staining for confocal analysis. The 721.221 cell line was stably transfected with a HLA-A*0201 cDNA in the pcDNA 3.1(-) vector (Invitrogen, Carlsbad, CA, USA) to generate the 721.221A2 cell line. The 721.221-A24 and 721.221-A2 cell lines were transfected, using the Amaxa Nucleofector, with either the pCMV-Tag4A vector or with APLP2-pCMV-Tag4A at 24 hours prior to flow cytometry analysis. The MDA-MB435S cell line was stably transfected with the pCMV-Tag4A vector or APLP2-pCMV-Tag4A by the use of Effectene (Qiagen, Inc., Valencia, CA, USA). The GFP-tagged Rab5 and Rab11 cDNAs $(17,47)$ in the EGFP vector (Clontech, Mountain View, CA, USA) used in transient transfections to identify early endosomes and recycling endosomes, respectively $(8,15,34)$, were gifts from Dr. R. Lodge. To ascertain if the perinuclear compartment in which HLA class I and APLP2 co-localized was the Golgi, a YFP-Golgi construct (Clontech, Mountain View, CA, USA) was transiently transfected into the cells. All transfections were performed using $1 \mu \mathrm{g}$ DNA per $0.5 \times 10^{6}$ cells.

\section{Immunoprecipitations and Western blots}

The immunoprecipitations and Western blots were done by procedures similar to published methods (53). For immunoprecipitations from radiolabeled cell lysates, cells were first grown in methionine- and cysteine-free medium for $30 \mathrm{~min}$ and then radiolabeled with $\left[{ }^{35} \mathrm{~S}\right]-$ methionine/cysteine for $30 \mathrm{~min}$. The labeled cells were washed 3 times with $20 \mathrm{mM}$ iodoacetamide (Sigma-Aldrich, St. Louis, MO, USA) in PBS. The cells were lysed with $1 \%$ 3-[(3-cholamidopropyl) dimethylammonio]-1-propanesulfonate (CHAPS) in Tris-buffered saline ( $\mathrm{pH}$ 7.4) containing $20 \mathrm{mM}$ iodoacetamide and freshly added $0.2 \mathrm{mM}$ PMSF and excess antibody, and the lysates were incubated on ice for 1 hour before centrifugation. The clarified lysates were incubated with Protein A-Sepharose beads (Amersham Biosciences, Piscataway, 
NJ, USA) for 45 minutes. The beads were washed 4 times with 0.1\% CHAPS in TBS (pH 7.4) and then the immunoprecipitated proteins were eluted from the beads by boiling in $50 \mu \mathrm{l}$ of $0.125 \mathrm{M}$ Tris ( $\mathrm{pH}$ 6.8)/2\% SDS/12\% glycerol/0.02\% bromophenol blue (1X elution buffer) for 5 minutes and loaded onto precast $4 \rightarrow 20 \%$ acrylamide Tris-glycine gels (Invitrogen, Carlsbad, CA, USA). For autoradiography, following electrophoresis the proteins were transferred to Immobilon-P membranes (Millipore, Billerica, MA, USA), which were subsequently dried and exposed to Kodak BioMax film (Eastman Kodak Co., Rochester, NY, USA). For immunoprecipitations from non-radiolabeled cells, the cells were washed in $20 \mathrm{mM}$ iodoacetamide in PBS 3 times and then treated with CHAPS lysis buffer. Subsequent steps were as described above, except that the membranes were developed as Western blots instead of being dried and exposed to film.

For Western blotting, immunoprecipitated proteins were electrophoresed on precast $4 \rightarrow 20 \%$ acrylamide Tris-glycine gels and the proteins were then transferred to Immobilon-P blotting membranes (Millipore, Bedford, MA, USA). After blocking overnight in reconstituted dry milk, each blotting membrane was incubated in primary antibody diluted in milk for 2 hours, washed 3 times with $0.05 \%$ Tween 20/PBS, and incubated for 1 hour in biotin-conjugated goat anti-mouse or anti-rabbit IgG (Caltag Laboratories, San Francisco, CA, USA) diluted 1:2,000 in $0.05 \%$ Tween 20/PBS. After 3 washes with $0.05 \%$ Tween 20/PBS, the membranes were incubated for 1 hour with streptavidin-conjugated horseradish peroxidase (Zymed, San Francisco, CA, USA) that had been diluted 1:20,000 in 0.05\% Tween 20/PBS. The membranes were then washed with $0.3 \%$ Tween 20/PBS 3 times, and treated with enhanced chemiluminescence Western blot developing reagents (Amersham Biosciences, Piscataway, NJ, USA). The blots were exposed to film to document the bands.

When Western blots were performed on cell lysates without an immunoprecipitation step, the samples were processed prior to electrophoresis as follows. The cells were washed 3 times in PBS with $20 \mathrm{mM}$ iodoacetamide and lysed in $1 \mathrm{X}$ elution buffer with freshly added $0.2 \mathrm{mM}$ PMSF. The lysates were incubated 1 hour on ice and were centrifuged to pellet nuclear material. Samples of the supernatants were boiled before loading onto gels, and blotting and developing was performed as described above.

\section{Immunofluorescence analysis}

To assess APLP2/HLA class I interaction at steady state, S2-013 cells were grown on glass cover slips and fixed with $4 \%$ (vol/vol) paraformaldehyde in PBS for 10 minutes. Fixed cells were incubated with mouse anti-HLA-A,B,C antibody and rabbit anti-APLP2 antibody prepared in staining solution [0.2\% saponin (wt/vol) and $0.5 \%(\mathrm{wt} / \mathrm{vol})$ bovine serum albumin in PBS] for 1 hour at room temperature. After 3 PBS washes (5 minutes/wash), the cells were incubated with fluorochrome-conjugated secondary antibodies (Alexa Fluor 568 goat antimouse and Alexa Fluor 488 goat anti-rabbit antibodies) in staining solution for 30 minutes at room temperature. After 3 washes in PBS (5 minutes/wash), the cells were mounted for confocal image analysis.

To test for APLP2 interaction with HLA molecules endocytosed from the plasma membrane, S2-013 cells were grown on glass cover slips, treated with anti-HLA-A,B,C antibody at $4{ }^{\circ} \mathrm{C}$, and incubated at $37^{\circ} \mathrm{C}$ for 30 minutes. At the end of the pulse period, remaining antibody bound to cell surface HLA class I molecules was removed by incubation of the cells in stripping buffer $(0.5 \%$ acetic acid, $500 \mathrm{mM} \mathrm{NaCl})$ for 90 seconds and then the cells were fixed with $4 \%$ paraformaldehyde in PBS for 10 minutes. After fixation, cells were incubated with rabbit antiAPLP2 antibody in staining solution for 1 hour at room temperature, and washed 3 times (5 minutes/wash) with PBS. The cells were then incubated with Alexa Fluor 488 goat anti-rabbit antibody and Alexa Fluor 568 goat anti-mouse antibody in staining solution for 30 minutes at room temperature, washed again 3 times with PBS (5 minutes/wash), and mounted for image 
analysis. Immunofluorescence analysis was also used to show lack of co-localization between APLP2 and transferrin receptor, as well as to demonstrate the co-localization of early endosomal markers Rab5 and EEA1 and the recycling endosome marker Rab11 with APLP2 and endocytosed HLA class I molecules. The full methodological details for each of these specific experiments are provided in the relevant figure legends.

To analyze changes in the localization of HLA-A24 molecules in HeLa cells that expressed an increased level of APLP2, HeLa cells stably expressing HLA-A*2402 were cultured on glass cover slips and transfected with APLP2-FLAG using Effectene. At 24 hours post-transfection, the cells were fixed with $4 \%$ paraformaldehyde in PBS for 10 minutes. Fixed cells were incubated with a monoclonal antibody that recognizes HLA-A24 and a rabbit antiserum against the FLAG tag in staining solution for 1 hour at room temperature. After 3 washes (5 minutes each) in PBS, the cells were incubated with a mixture of fluorochrome-labeled secondary antibodies (Alexa Fluor 568 goat anti-mouse antibody and Alexa Fluor 488 goat anti-rabbit antibody) in staining solution for 30 minutes at room temperature, and then washed again 3 times (5 minutes each) in PBS. Immunofluorescence analysis was also used to show the colocalization of a Golgi marker with APLP2 and HLA-A*2402, and to demonstrate the colocalization of APLP2 and endocytosed HLA-A*2402 class I molecules in vesicles containing the early endosomal marker EEA1. The complete technical procedures for each of these specific experiments are included in the appropriate figure legends.

\section{Flow cytometry}

For the flow cytometry assays, cells were first suspended at $5 \times 10^{6} / \mathrm{ml}$ in PBS containing $0.2 \%$ BSA and $0.1 \%$ sodium azide. Cell suspension aliquots $(0.1 \mathrm{ml}$ each) were distributed into the wells of a 96-well plate. The cells were incubated with excess antibody or with only PBS+BSA/ azide at $4^{\circ} \mathrm{C}$ for 30 minutes, washed twice, and incubated with a phycoerythrin-conjugated, $\mathrm{Fc}$-specific $\mathrm{F}(\mathrm{ab} \text { ') })_{2}$ portion of goat anti-mouse IgG (Jackson ImmunoResearch Laboratories, Inc., West Grove, PA, USA) for 30 minutes at $4^{\circ} \mathrm{C}$. The cells were washed 3 times, resuspended in PBS+BSA/azide, and analyzed with a FACSCalibur flow cytometer and Cell Quest software (BD Biosciences, San Jose, CA, USA).

\section{Results}

\section{Endogenous APLP2 molecules were found to associate with endogenous HLA class I molecules in intracellular vesicles of human tumor cells}

To begin our examination of the interaction of APLP2 with HLA class I molecules, we assessed the levels of APLP2 expressed by various human cell lines by Western blotting and normalized the results to beta-actin expression levels in the cell lines. As shown in Figure 1, the human tumor cell lines expressed divergent levels of APLP2 protein, ranging from high levels of APLP2 for the pancreatic tumor cell lines (Hs766T, S2-013 and SUIT2) to low levels in the lymphoma cell lines (SU-DHL-6 and CL-01). Results similar to those shown in the figure were obtained in three separate experiments. A Ewing's tumor cell line (A673) and a hepatoma cell line (Huh7) was also examined for APLP2 expression and found to express levels of APLP2 slightly lower than the breast carcinoma MDA-MB435S (data not shown). Thus, there was a wide range among the tumor cell lines in regard to APLP2 expression. The pancreatic tumor cell line S2-013, which has high APLP2 expression, was chosen for subsequent examination of APLP2/HLA class I co-localization.

To learn whether APLP2 and HLA class I molecules co-localized within human tumor cells, we used immunofluorescence. By permeabilizing S2-013 cells and staining for HLA class I molecules and APLP2, we demonstrated that APLP2 co-localized substantially with HLA class I molecules in vesicular compartments in these cells (see insets and arrows in Figure 2A). To 
examine whether APLP2 co-localized with endocytosed HLA class I molecules, cell surface HLA class I molecules on S2-013 cells were labeled with the anti-HLA-A,B,C antibody W6/32 and warmed to $37^{\circ} \mathrm{C}$. Upon internalization of W6/32-bound cell surface HLA class I molecules at $37^{\circ} \mathrm{C}$, we observed a substantial level of the endocytosed HLA class I molecules in APLP2containing vesicles (Figure 2B). In contrast to the extensive co-localization of endocytosed HLA class I molecules and APLP2, we observed relatively little co-localization of endocytosed transferrin receptor and APLP2 (Figure 2C). By the presence of Rab5 (Figure 3A) and EEA1 (Figure 3B), we identified many S2-013 vesicles in which HLA class I molecules and APLP2 were co-localized as early endosomes. These findings are consistent with our previous observation that $\mathrm{K}^{\mathrm{d}}$ molecules internalized from the cell surface are bound to APLP2 in Rab5positive early endosomes in HeLa cells (52). Furthermore, some of the S2-013 vesicles in which APLP2 and HLA class I molecules were both present could be characterized as recycling endosomes, as shown by the presence of Rab11 (Figure 4).

\section{Endogenous APLP2 was demonstrated to bind to the endogenous HLA class I molecule in human tumor cells}

To determine whether APLP2 bound to HLA class I molecules, we immunoprecipitated the HLA class I molecules from lysates of S2-013, MDA-MB435S, Hs766T, Huh7, and DU145 with W6/32. Our HLA typing of S2-013 and MDA-MB435S had shown that both cell lines expressed the HLA-A*2402 allele, and for those two cell lines we also performed immunoprecipitations with an anti-A24 antibody. By Western blotting, co-immunoprecipitated APLP2 was found associated to varied degrees with tumor cells' HLA molecules immunoprecipitated by W6/32 and anti-A24 antibody (Figure 5A). By comparison, immunoprecipitation of the transferrin receptor resulted in little co-immunoprecipitation of APLP2 (Figure 5B). As a control, efficient immunoprecipitation of the transferrin receptor was verified by using the anti-transferrin receptor antibody to probe a Western blot of the same immunoprecipitates (data not shown).

HLA typing of S2-013 cells had also revealed that S2-013 cells expressed HLA-A*0206. To determine whether APLP2 bound equally well to A2 or A24, immunoprecipitations were done on lysates of ${ }^{35} \mathrm{~S}$-methionine/cysteine-labeled S2-013 cells with antibodies recognizing HLAA2 or HLA-A24 (and the W6/32 antibody and anti-APLP2 serum as positive controls). (HLA$A^{*} 2402$ and HLA-A*0206 radiolabel very similarly with ${ }^{35}$ S-methionine/cysteine, since they have nearly identical numbers of methionines and cysteines: 5 cysteines each, 9 methionines in $\mathrm{A} * 2402$, and 8 methionines in $\mathrm{A} * 0206$, according to the IMGT/HLA internet site http://www.ebi.ac.uk/imgt/hla/.) The radiolabeled immunoprecipitates were electrophoresed and transferred to a membrane, which was then autoradiographed (Figure 5C, upper panel). Faint bands of the correct molecular weight to be APLP2 were present on the autoradiograph in the W6/32, anti-A24, and anti-A2 lanes, suggesting APLP2 co-immunoprecipitated with the HLA class I molecules (Figure 5C, upper panel). Some relatively light, diffuse, large molecular weight $(>200 \mathrm{kD})$ bands were also detected on the autoradiograph, especially in the anti-

APLP2, W6/32, and anti-A2 antibody lanes (data not shown), consistent with a previous report of high molecular weight glycosaminoglycan-modified APLP2 forms associated with $\mathrm{H} 2-\mathrm{K}^{\mathrm{d}}$ (39). The immunoprecipitates were also probed on a Western blot with anti-APLP2 serum to verify the position of APLP2 (Figure 5C, bottom panel). The Western blotting data indicated that APLP2 bound both to HLA-A2 and -A24, and to HLA-A2 slightly more strongly than to HLA-A24 (Figure 5C, bottom panel).

\section{Cell surface expression of transfected and endogenous HLA class I molecules is decreased by APLP2}

In previous experiments with the mouse MHC class I molecule $\mathrm{H} 2-\mathrm{K}^{\mathrm{d}}$, we demonstrated that the level of cellular APLP2 expression influenced $\mathrm{H} 2-\mathrm{K}^{\mathrm{d}}$ cell surface expression $(24,51,52)$. 
To test whether APLP2 affects the cell surface expression of human MHC class I molecules, we examined APLP2's effect on HLA-A2 and -A24, since we had ascertained these HLA molecules bind to APLP2 (as shown in Figure 5C). We transfected APLP2 into 721.221 cells expressing HLA-A2 or HLA-A24. The 721.221 cell line is a B lymphoblastoid cell line reported to lack endogenous HLA-A, B, and C molecules $(41,42)$, and therefore 721.221 cells transfected with a single HLA class I allele have been used as a model to study effects on a specific HLA class I molecule. Like the B lymphoma cell lines that we have tested (Figure 1), 721.221 expresses a very low level of total endogenous APLP2 (51). We found that increased expression of APLP2 substantially lowers the level of HLA-A2 and HLA-A24 at the plasma membrane of these cells, reducing their expression by $\sim 24-34 \%$ (Figure 6A). Similar results were obtained by transfecting APLP2 into 721.221-A24 cells in a vector also expressing GFP, and gating on GFP-expressing cells in the flow cytometry analysis (data not shown). Transfection of APLP2 into breast carcinoma MDA-MB435S cells also resulted in a lower level of cell surface total $\left(\mathrm{W} 6 / 32^{+}\right.$) HLA class I molecules and HLA-A24 (which is endogenously expressed by MDA-MB435S) (Figure 6B). Thus, the level of APLP2 expressed by a human tumor cell line can affect the surface expression of HLA-A2 and -A24 molecules.

Using confocal microscopy, we also analyzed the effect of APLP2 on the surface expression of transfected HLA-A24 on HeLa cells (Figure 7A). In HeLa cells, co-localization of APLP2 and HLA-A24 could be seen in some small vesicular structures, as displayed in the Figure 7B inset (note the magenta staining of two of the vesicles), and greater co-localization was observed in a compact perinuclear cellular compartment (Figure 7A and B). Consistent with our previous studies demonstrating that APLP2 and $\mathrm{K}^{\mathrm{d}}$ co-localize in the Golgi of HeLa cells (51), the compact compartment in which APLP2 and HLA-A24 co-localized in HeLa cells was also identified as the Golgi complex (Figure 7B). In the absence of additional (transfected) APLP2, staining of HLA-A*2402 was intense at the outer edges of the cells (e.g., see the area indicated by an arrow on the upper left side of the first and third panels of Figure 7A). In cells that were transfected with APLP2-FLAG, there was a visible reduction in the amount of HLA$A * 2402$ at or near the plasma membrane (e.g., see the area indicated by the arrow on the upper right side of the first and third panels of Figure 7A, and compare the area indicated by the upper arrow to the area indicated by the lower arrow in Figure 7B).

We also examined by confocal microscopy whether APLP2 co-localized with endocytosed HLA-A*2402 in HeLa cells expressing A*2402. Cell surface A*2402 molecules were labeled with anti-A24 antibody and the cells were incubated at $37^{\circ} \mathrm{C}$. Following the internalization of the labeled molecules, we detected endocytosed A*2402 molecules co-localized with APLP2 in vesicles (Figure 8A) that were identifiable as early endosomes by the presence of EEA1 (Figure 8B). Thus, when HLA-A*2402 endocytosis was induced, the presence of APLP2 in the endocytic vesicles bearing $A * 2402$ could be clearly observed.

\section{Discussion}

In this study, we demonstrated that elevated levels of APLP2 (due to APLP2 transfection) reduced the cell surface expression of endogenous, total (W6/32+) HLA class I molecules and the endogenous HLA-A24 allotype on MDA-MB435S tumor cells. We also over expressed APLP2 in the B lymphoblastoid cell line 721.221 transfected with HLA-A2 or -A24, and showed that HLA-A2 and -A24 expression at the plasma membrane decreased. Our discovery that increased expression of APLP2 reduced surface HLA class I suggests that high APLP2 expression in cancer might aid escape from immunosurveillance, since elevated APLP2 has been reported for some tumors $(1,10)$.

We also attempted to determine whether APLP2-specific siRNA expressed in the pancreatic tumor cell lines S2-013 and Hs766T would cause an increase in surface HLA class I, but down 
regulation of APLP2 expression in S2-013 and Hs766T stopped proliferation of these cells in culture, and therefore MHC class I cell expression at the plasma membrane might be affected secondarily by the cellular stress and changes in protein synthesis, transport, and turnover that likely would accompany such a rapid decrease in cell proliferation. For S2-013, we quantified this decrease in proliferation by MTT assay, and found that there was a 38\% decrease in proliferation with APLP2 siRNA transfection (compared to control siRNA transfection) by 72 hours after transient transfection. Hs766T cells transfected with APLP2 siRNA also had slowed proliferation relative to cells transfected with control siRNA (data not shown). Since APLP2 has been shown to affect cytokinesis in some cells (32), a disruption in cell cycle control may have resulted from the transfection of APLP2 siRNA into these cells. In contrast, in our earlier experiments done with HeLa cells, HeLa cell proliferation was unaffected by either transient or stable down regulation of APLP2 expression (52 and data not shown). Evidently the influence of APLP2 on cellular proliferation varies depending on the cell type.

By flow cytometric analysis, we evaluated the levels of MHC class I molecules on the surfaces of all the tumor cell lines for which we had APLP2 expression data from Western blots (Figure 1 and data not shown). We did not find consistent correlation between APLP2 expression and surface MHC class I levels throughout the panel of tumor cell lines. This lack of correlation may be because many factors regulate MHC class I surface expression. In this case, such factors include the type of tumor (pancreatic tumor, breast tumor, and so forth), the levels of the proteins known to be directly involved in MHC class I assembly (such as tapasin and the transporter associated with antigen processing, which have been shown to be down regulated in some tumors), and the levels of other cellular proteins that indirectly affect MHC class I or APLP2 $(4,21,37)$.

We found APLP2 was co-localized with folded HLA class I molecules by examining the cellular distribution of the molecules following endocytosis of HLA class I molecules from the cell surface. Evidence has been presented for MHC class I endocytosis by clathrinindependent mechanisms (31) and by clathrin-coated pits (11), and MHC class I molecules expressed by B lymphoblastoid cells have been demonstrated to be continually endocytosed and recycled to the cell surface (33). MHC class I recycling is dependent on the Eps15 homology domain-containing protein (EHD) 1 , which induces the generation of tubules that hold internalized MHC class I molecules $(5,27)$. An ortholog of EHD1 (EHD4) has also been shown to influence the recycling of MHC class I molecules (40). The co-localization of APLP2 with endocytosed HLA class I molecules raises the possibility that APLP2 may influence HLA class I recycling and/or degradation in tumor cells. At this point, we do not know what other endocytosed proteins, if any, in addition to MHC class I molecules may co-localize in endocytic vesicles with APLP2, but these findings do suggest that APLP2 preferentially co-localizes with endocytosed MHC class I molecules relative to at least one other protein that undergoes endocytosis (i.e., the transferrin receptor).

Overall, our study has provided new insight into the regulation of HLA class I molecule cell surface expression. Our findings have shown that human tumor cell lines express APLP2 in varied amounts, and that APLP2 binds HLA class I molecules in human tumor cell lines. HLA class I molecules internalized from the cell surface were found co-localized with APLP2 in vesicles, suggesting that APLP2 may be involved in regulating trafficking and turnover of HLA class I molecules. Furthermore, our results indicate that the amount of APLP2 expressed in cells affects the level of HLA class I molecules at the plasma membrane. In total, these data suggest that APLP2 may play an important role in the regulation of antigen presentation by HLA class I molecules. 


\section{Acknowledgments}

We thank Dr. Shantaram Joshi, Dr. Kenneth Cowan, Dr. Vinod Labhasetwar, Dr. Michael A. Hollingsworth, Dr. Richard MacDonald, Dr. John Chan, Vivek Gautam, Christopher Connelly, Michelle Hartman, Himabindu Ramachandrareddy, Jaspreet Vasir, Tom Caffrey, Dr. Pankaj Singh, Dr. Wendy Maury, and Dr. Ted Hansen for their assistance with obtaining cell lines and antibodies, and we thank Dr. Atsunori Hiasa and Dr. Hiroshi Shiku for the HLA-A*2404 cDNA. We gratefully acknowledge the assistance of the personnel of the UNMC Cell Analysis Facility and the Monoclonal Antibody Facility. Core facilities at UNMC receive support from the NIH Cancer Center Support Grant P30CA036727. This work was supported by NIH Grants GM57428 (to J.C.S.) and GM74876 (to S.C.), an Eppley Cancer Center Pediatric Cancer Research Grant, UNMC Graduate Studies Fellowships (to A.T., M.S., and L.S.), a Nebraska Center for Cellular Signaling Fellowship and an American Heart Association Predoctoral Fellowship (to M.S.), an NIH Training Grant T32 CA009476 Fellowship (to L.S.), and a Graduate Assistance in Areas of National Need Fellowship (to H.C.).

\section{References}

1. Abba MC, Drake JA, Hawkins KA, Hu Y, Sun H, Notcovich C, Gaddis S, Sahin A, Baggerly K, Marcelo Aldaz C. Transcriptomic changes in human breast cancer progression as determined by serial analysis of gene expression. Breast Cancer Res 2004;6:499-513.

2. Anliker B, Müller U. The functions of mammalian amyloid precursor protein and related amyloid precursor-like proteins. Neurodegener Dis 2006;3:239-246. [PubMed: 17047363]

3. Cailleau R, Olive M, Cruciger OV. Long-term human breast carcinoma cell lines of metastatic origin: preliminary characterization. In Vitro 1978;17:911-915. [PubMed: 730202]

4. Campoli M, Ferrone S. HLA antigen changes in malignant cells: epigenetic mechanisms and biologic significance. Oncogene 2008;27:5869-5885. [PubMed: 18836468]

5. Caplan S, Naslavsky N, Hartnell LM, Lodge R, Polishchuk RS, Donaldson JG, Bonifacino JS. A tubular EHD1-containing compartment involved in the recycling of major histocompatibility complex class I molecules to the plasma membrane. EMBO J 2002;21:2557-2567. [PubMed: 12032069]

6. Cappai R, Mok SS, Galatis D, Tucker DF, Henry A, Beyreuther K, Small DH, Masters CL. Recombinant human amyloid precursor-like protein 2 (APLP2) expressed in the yeast Pichia pastoris can stimulate neurite outgrowth. FEBS Lett 1999;442:95-98. [PubMed: 9923612]

7. Carreno BM, Hansen TH. Exogenous peptide ligand influences the expression and half-life of free HLA class I heavy chains ubiquitously detected at the cell surface. Eur J Immunol 1994;24:12851292. [PubMed: 8206088]

8. Casanova JE, Wang X, Kumar R, Bhartur SG, Navarre J, Woodrum JE, Altschuler Y, Ray GS, Goldenring JR. Association of Rab25 and Rab11a with the apical recycling system of polarized MadinDarby canine kidney cells. Mol Biol Cell 1999;10:47-61. [PubMed: 9880326]

9. Cerutti A, Zan H, Schaffer A, Bergsagel L, Harindranath N, Max EE, Casali P. CD40 ligand and appropriate cytokines induce switching to $\mathrm{IgG}, \mathrm{IgA}$, and $\mathrm{IgE}$ and coordinated germinal center and plasmacytoid phenotypic differentiation in a human monoclonal $\operatorname{IgM}+\operatorname{IgD}+\mathrm{B}$ cell line. J Immunol 1998;160:2145-2157. [PubMed: 9498752]

10. Covell DG, Wallqvist A, Rabow AA, Thanki N. Molecular classification of cancer: unsupervised self-organizing map analysis of gene expression microarray data. Mol Cancer Therap 2003;2:317332. [PubMed: 12657727]

11. Dasgupta JD, Watkins S, Slayter H, Yunis EJ. Receptor-like nature of class I HLA: endocytosis via coated pits. J Immunol 1988;141:2577-2580. [PubMed: 2902138]

12. Epstein AL, Levy R, Kim H, Henle W, Henle G, Kaplan HS. Biology of the human malignant lymphomas IV: Functional characterization of ten diffuse histiocytic lymphoma cell lines. Cancer 1978;42:2379-2391. [PubMed: 214220]

13. Feuerbach D, Burgert H-G. Novel proteins associated with MHC class I antigens in cells expressing adenovirus protein E3/19K. EMBO J 1993;12:3153-3161. [PubMed: 8344254]

14. Foung SKH, Taidi B, Ness D, Grumet FC. A monoclonal antibody against HLA-A11 and A24. Human Immunol 1986;15:316-319. [PubMed: 2420768]

15. Gorvel JP, Chavrier P, Zerial M, Gruenberg J. Rab5 controls early endosome fusion in vitro. Cell 1991;64:915-925. [PubMed: 1900457](1991) 
16. Guo J, Thinakaran G, Guo Y, Sisodia SS, Yu FX. A role for amyloid precursor-like protein 2 in corneal epithelial wound healing. Invest Ophthalmol Vis Sci 1998;39:292-300. [PubMed: 9477985]

17. Hunyady L, Baukal AJ, Gaborik Z, Olivares-Reyes JA, Bor M, Szaszak M, Lodge R, Catt KJ, Balla T. Differential PI 3-kinase dependence of early and late phases of recycling of the internalized $\mathrm{AT}_{1}$ angiotensin receptor. J Cell Biol 2002;157:1211-1222. [PubMed: 12070129]

18. Iwamura T, Katsuki T, Ide K. Establishment and characterization of a human pancreatic cancer cell line (SUIT-2) producing carcinoembryonic antigen and carbohydrate antigen 19-9. Jpn J Cancer Res 1987;78:54-62. [PubMed: 3102439]

19. Ladasky JJ, Shum BP, Canavez F, Seuanez HN, Parham P. Residue 3 of beta2-microglobulin affects binding of class I MHC molecules by the W6/32 antibody. Immunogenetics 1999;49:312-320. [PubMed: 10079295]

20. Li XF, Thinakaran G, Sisodia SS, Yu FS. Amyloid precursor-like protein 2 promotes cell migration toward fibronectin and collagen IV. J Biol Chem 1999;274:27249-27256. [PubMed: 10480944]

21. Lopez-Albaitero A, Navak JV, Ogino T, Machandia A, Gooding W, DeLeo AB, Ferrone S, Ferris RL. Role of antigen-processing machinery in the in vitro resistance of squamous cell carcinoma of the head and neck cells to recognition by CTL. J Immunol 2006;176:3402-3409. [PubMed: 16517708]

22. Martinez-Ramirez A, Rodriguez-Perales S, Melendez B, Martinez-Delgado B, Urioste M, Cigudosa JC, Benitez J. Characterization of the A673 cell line (Ewing tumor) by molecular cytogenetic techniques. Cancer Genet Cytogenet 2003;141:138-142. [PubMed: 12606131]

23. Miyahara Y, Naota H, Wang L, Hiasa A, Goto M, Watanabe M, Kitano S, Okumura S, Takemitsu T, Yuta A, Majima Y, Lemonnier FA, Boon T, Shiku H. Determination of cellularly processed HLAA2402-restricted novel CTL epitopes derived from two cancer germ line genes, MAGE-A4 and SAGE. Clin Cancer Res 2005;11:5581-5589. [PubMed: 16061876]

24. Morris CR, Petersen JL, Vargas SE, Turnquist HR, McIlhaney MM, Sanderson SD, Bruder JT, Yu YYL, Burgert H-G, Solheim JC. The amyloid precursor-like protein 2 and the adenoviral E3/19K protein both bind to a conformational site on $\mathrm{H}-2 \mathrm{~K}^{\mathrm{d}}$ and regulate $\mathrm{H}-2 \mathrm{~K}^{\mathrm{d}}$ expression. $\mathrm{J}$ Biol Chem 2003;278:12618-12623. [PubMed: 12506118]

25. Mu FT, Callaghan JM, Steele-Mortimer O, Stenmark H, Parton RG, Campbell PL, McCluskey J, Yeo JP, Tock EP, Toh BH. EEA1, an early endosome-associated protein: EEA1 is a conserved alphahelical peripheral membrane protein flanked by cysteine "finger" and contains a calmodulin-binding IQ motif. J Biol Chem 1995;270:133503-133511.

26. Nakabayashi H, Taketa K, Miyano K, Yamane T, Sato J. Growth of human hepatoma cell lines with differentiated functions in chemically defined medium. Cancer Res 1982;42:3858-3863. [PubMed: 6286115]

27. Naslavsky N, Boehm M, Backlund PS Jr, Caplan S. Rabenosyn-5 and EHD1 interact and sequentially regulate protein recycling to the plasma membrane. Mol Biol Cell 2004;15:2410-2422. [PubMed: 15020713]

28. Owens RB, Smith HS, Nelson-Rees WA, Springer EL. Epithelial cell cultures from normal and cancerous human tissues. J Natl Cancer Inst 1976;56:843-849. [PubMed: 176412]

29. Parham P, Barnstable CJ, Bodmer WF. Use of a monoclonal antibody (W6/32) in structural studies of HLA-A,B,C antigens. J Immunol 1979;123:342-349. [PubMed: 87477]

30. Parham P, Brodsky FM. Partial purification and some properties of BB7.2: a cytotoxic monoclonal antibody with specificity for HLA-A2 and a variant of HLA-A28. 1981

31. Radhakrishna H, Donaldson JG. ADP-ribosylation factor 6 regulates a novel plasma membrane recycling pathway. J Cell Biol 1997;139:49-61. [PubMed: 9314528]

32. Rassoulzadegan M, Yang Y, Cuzin F. APLP2, a member of the Alzheimer precursor protein family, is required for correct genomic segregation in dividing mouse cells. EMBO J 1998;17:4647-4656. [PubMed: 9707424]

33. Reid PA, Watts C. Cycling of cell-surface MHC glycoproteins through primaquine-sensitive intracellular compartments. Nature 1990;346:655-657. [PubMed: 2166918]

34. Ren M, Xu G, Zeng J, De Lemos-Chiarandini C, Adesnik M, Sabatini DD. Hydrolysis of GTP on rab11 is required for the direct delivery of transferrin from the pericentriolar recycling compartment 
to the cell surface but not from sorting endosomes. Proc Natl Acad Sci USA 1998;95:6187-6192. [PubMed: 9600939]

35. Scheinfeld MH, Ghersi E, Laky K, Fowlkes BJ, D'Adamio L. Processing of beta-amyloid precursorlike protein-1 and -2 by gamma-secretase regulates transcription. J Biol Chem 2002;277:4419544201. [PubMed: 12228233]

36. Scherer WF, Syverton JT, Gey GO. Studies on the propagation in vitro of poliomyelitis viruses. IV. Viral multiplication in a stable strain of human malignant epithelial cells (strain HeLa) derived from an epidermoid carcinoma of the cervix. J Exp Med 1953;97:695-710. [PubMed: 13052828]

37. Seliger B, Ritz U, Ferrone S. Molecular mechanisms of HLA class I antigen abnormalities following viral infection and transformation. Int J Cancer 2006;118:129-138. [PubMed: 16003759]

38. Sernee MF, Ploegh HL, Schust DJ. Why certain antibodies cross-react with HLA-A and HLA-G: epitope mapping of two common MHC class I reagents. Mol Immunol 1998;35:177-188. [PubMed: 9694518]

39. Sester M, Feuerbach D, Frank R, Preckel T, Gutermann A, Burgert H-G. The amyloid precursor-like protein 2 associates with the major histocompatibility complex class I molecule $\mathrm{K}^{\mathrm{d}}$. J Biol Chem 2000;275:3645-3654. [PubMed: 10652361]

40. Sharma M, Naslavsky N, Caplan S. A role for EHD4 in the regulation of early endosomal transport. 2008Traffic, epub

41. Shimizu Y, Geraghty DE, Koller BH, Orr HT, DeMars R. Transfer and expression of three cloned human non-HLA-A,B,C class I major histocompatibility complex genes in mutant lymphoblastoid cells. Proc Natl Acad Sci USA 1988;85:227-231. [PubMed: 3257565]

42. Shimizu Y, DeMars R. Production of human cells expressing individual transferred HLA-A,-B,-C genes using an HLA-A,-B,-C null human cell line. J Immunol 1989;142:3320-3328. [PubMed: 2785140]

43. Slunt HH, Thinakaran G, Von Koch C, Lo ACY, Tanzi RE, Sisodia SS. Expression of a ubiquitous, cross-reactive homologue of the mouse $\beta$-amyloid precursor protein. J Biol Chem 1994;269:26372644. [PubMed: 8300594]

44. Soule HD, Vazquez J, Long A, Albert S, Brennan M. A human cell line from a pleural effusion derived from a breast carcinoma. J Natl Cancer Inst 1973;51:1409-1416. [PubMed: 4357757]

45. Stam N, Spits H, Ploegh H. Monoclonal antibodies raised against denatured HLA-B locus heavy chains permit biochemical characterization of certain HLA-C locus products. J Immunol 1986;137:2299-2306. [PubMed: 3760563]

46. Stam NJ, Vroom TM, Peters PJ, Pastoors EB, Ploegh HL. HLA-A- and HLA-B-specific monoclonal antibodies reactive with free heavy chains in western blots, in formalin-fixed, paraffin-embedded tissue sections and in cryo-immuno-electron microscopy. Int Immunol 1990;2:113-125. [PubMed: 2088481]

47. Stenmark H, Parton RG, Steele-Mortimer O, Lutcke A, Gruenberg J, Zerial M. Inhibition of rab5 GTPase activity stimulates membrane fusion in endocytosis. EMBO J 1994;13:1287-1296. [PubMed: 8137813]

48. Stone KR, Mickey DD, Wunderli H, Mickey GH, Paulson DF. Isolation of a human prostate carcinoma cell line (DU 145). Int J Cancer 1978;21:274-281. [PubMed: 631930]

49. Thinakaran G, Kitt CA, Roskams AJ, Slunt HH, Masliah E, von Koch C, Ginsberg SD, Ronnett GV, Reed RR, Price DL. Distribution of an APP homolog, APLP2, in the mouse olfactory system: a potential role for APLP2 in axogenesis. J Neurosci 1995;15:6314-6326. [PubMed: 7472397]

50. Tsutsumida H, Swanson BJ, Singh PK, Caffrey TC, Kitajima S, Goto M, Yonezawa S, Hollingsworth MA. RNA interference suppression of MUC1 reduces the growth rate and metastatic phenotype of human pancreatic cancer cells. Clin Cancer Res 2006;12:2976-2987. [PubMed: 16707592]

51. Tuli A, Sharma M, Wang X, Naslavsky N, Caplan S, Solheim JC. Specificity of amyloid precursorlike protein 2 interactions with MHC class I molecules. Immunogenetics 2008;60:303-313. [PubMed: 18452037]

52. Tuli A, Sharma M, McIlhaney MM, Talmadge JE, Naslavsky N, Caplan S, Solheim JC. Amyloid precursor-like protein 2 increases the endocytosis, instability, and turnover of the $\mathrm{H} 2-\mathrm{K}^{\mathrm{d}} \mathrm{MHC}$ class I molecule. J Immunol 2008;181:1978-1987. [PubMed: 18641335] 
53. Turnquist HR, Solheim JC. Analysis of MHC class I interactions with endoplasmic reticulum proteins. Methods Mol Biol 2001;156:165-173. [PubMed: 11068758]

54. Walsh DM, Fadeeva JV, Lavoie MJ, Paliga K, Egger S, Kimberly WT, Wasco W, Selkoe DJ. Gammasecretase cleavage and binding to FE65 regulate the nuclear translocation of the intracellular Cterminal domain (ICD) of the APP family of proteins. Biochemistry 2003;42:6664-6673. [PubMed: 12779321]

55. Walsh DM, Minogue AM, Frigerio CS, Fadeeva JV, Wasco W, Selkoe DJ. The APP family of proteins: similarities and differences. Biochem Soc Transac 2007;5:416-420.

56. Wang B, Yang L, Wang Z, Zheng H. Amyloid precursor protein mediates presynaptic localization and activity of the high-affinity choline transporter. Proc Natl Acad Sci USA 2007;104:14140-14145. [PubMed: 17709753] 


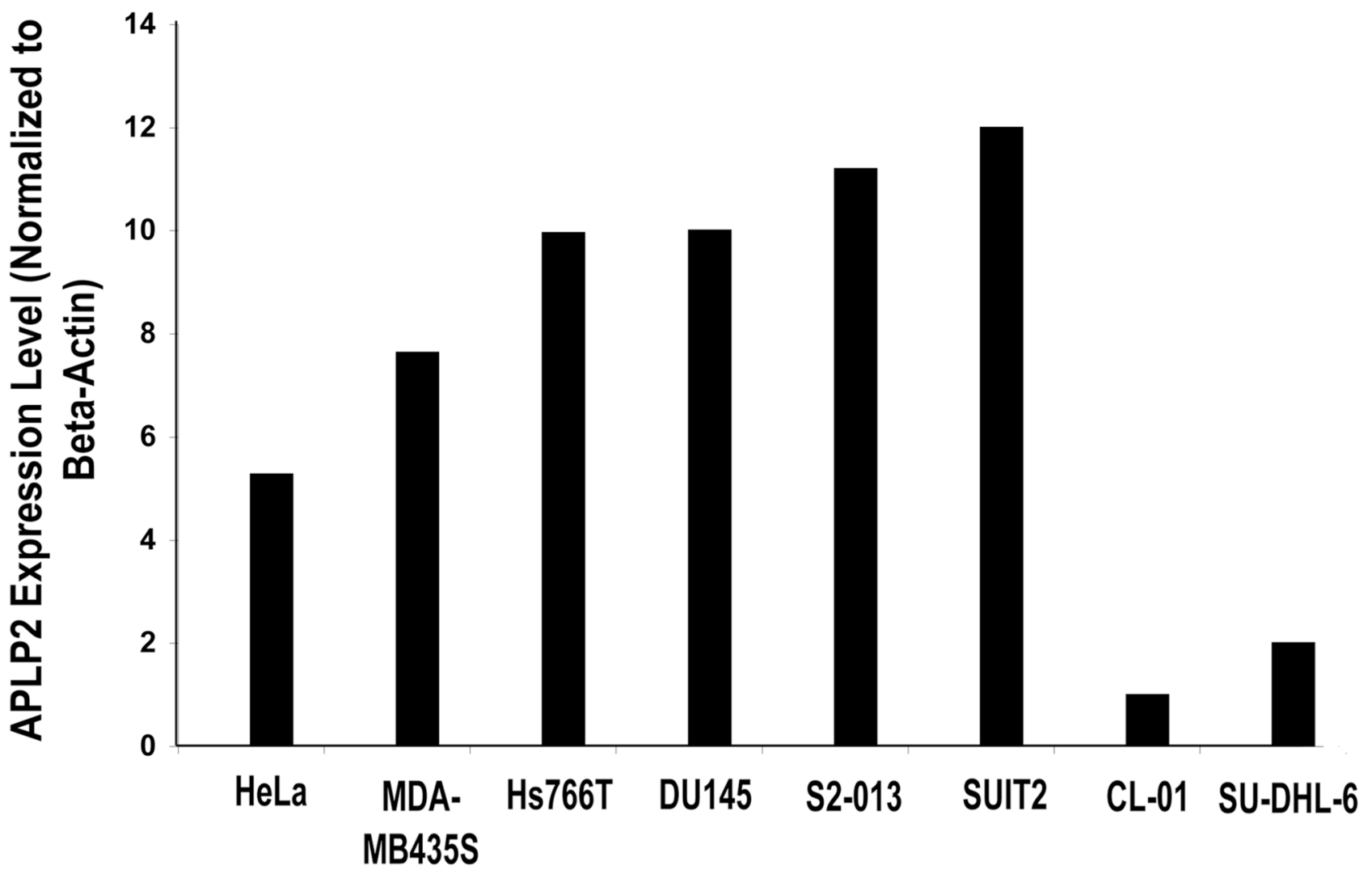

Cell Lines

Figure 1.

The expression of APLP2 in human tumor cell lines varied greatly among the cell lines tested. Samples of the indicated cell lysates (made from equal numbers of cells from each cell line) were electrophoresed on a 4-20\% Tris-glycine acrylamide gel, transferred to a Western blot, and probed with APLP2-specific antiserum for comparison of the total amount of APLP2 within the cells or with an antibody that recognizes $\beta$-actin. The results for APLP2 were normalized to the results for $\beta$-actin and the normalized results are displayed on the bar graph. The results shown are representative of the results obtained in three independent experiments. 

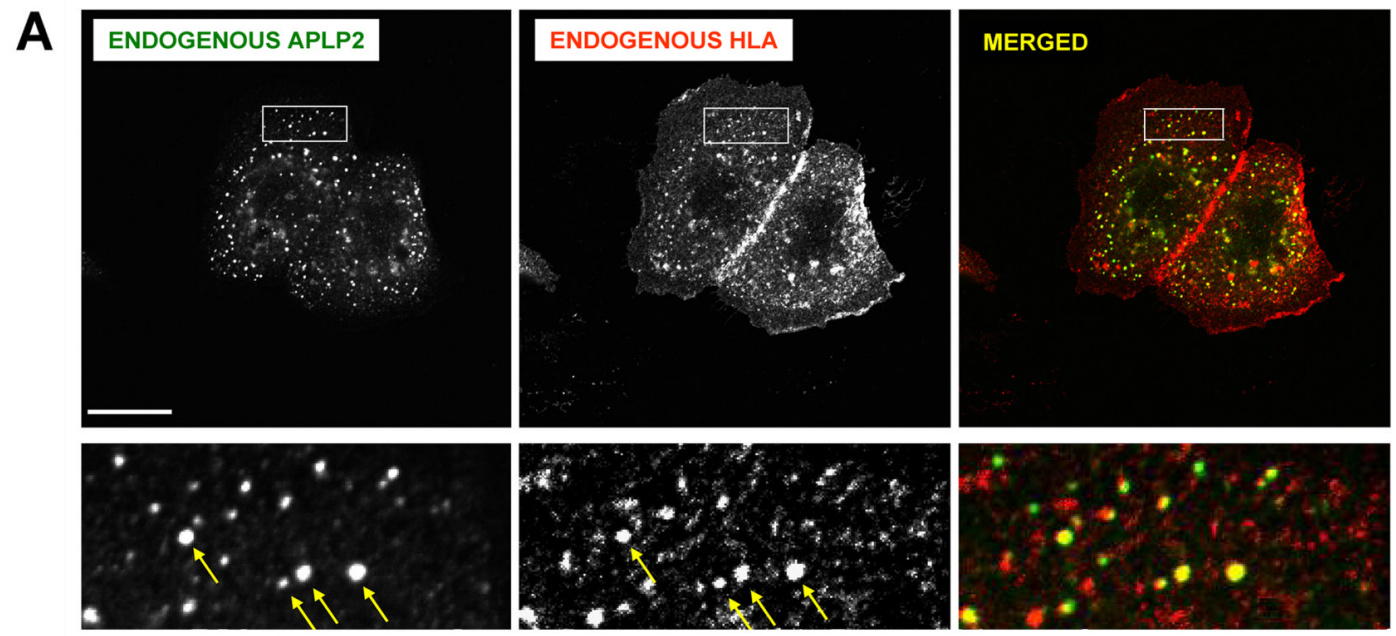

B
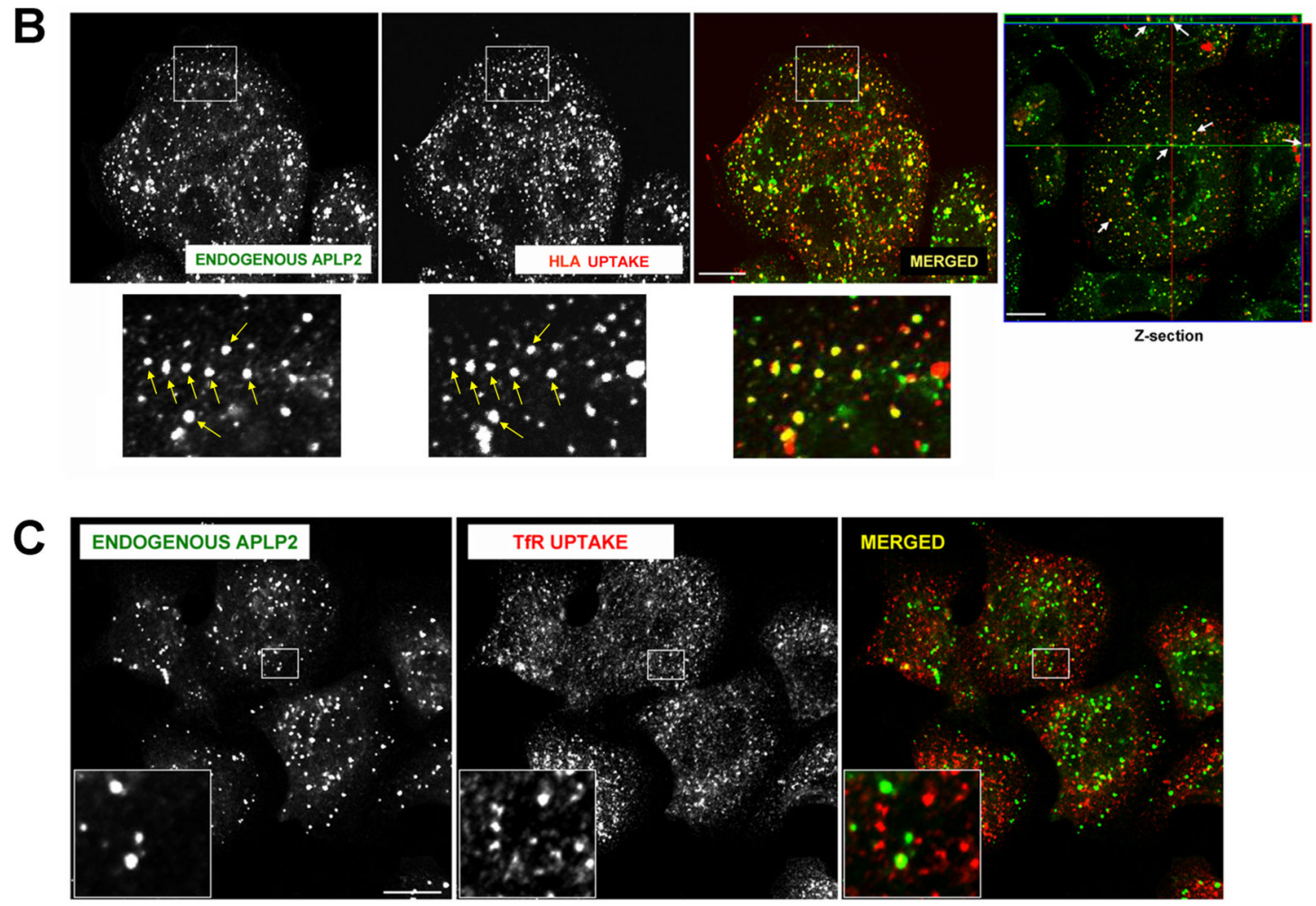

Figure 2.

APLP2 molecules were co-localized with folded HLA class I molecules in vesicular structures in human pancreatic tumor S2-013 cells. (A) S2-013 cells were fixed with 4\%

paraformaldehyde in PBS for 10 minutes prior to incubation in staining solution $(0.2 \%$ saponin, $0.5 \%$ bovine serum albumin in PBS) with mouse anti-HLA-A,B,C antibody (W6/32) and rabbit anti-APLP2 serum for 1 hour at room temperature. The cells were then washed 3 times with PBS (5 minutes/wash) and incubated with fluorescently labeled secondary antibodies (Alexa Fluor 568 goat anti-mouse and Alexa Fluor 488 goat anti-rabbit antibodies) for 30 minutes at room temperature. Green $=$ APLP2; red $=$ folded HLA class I molecules; yellow $=$ co-localized APLP2 and HLA class I molecules. Yellow arrows indicate vesicles containing co-localized 
endogenous HLA class I and APLP2 molecules. More highly magnified images of areas shown in the larger boxes are shown below the boxes. (B) S2-013 cells were incubated with mouse anti-HLA-A,B,C antibody (W6/32) at $4^{\circ} \mathrm{C}$, then warmed to $37^{\circ} \mathrm{C}$ for 30 minutes. The cells were treated with stripping solution $(0.5 \%$ acetic acid, $500 \mathrm{mM} \mathrm{NaCl})$ for 90 seconds to remove non-internalized surface-bound antibody, and fixed with 4\% paraformaldehyde in PBS for 10 minutes. The cells were then incubated in staining solution with rabbit anti-APLP2 serum for 1 hour at room temperature, washed 3 times (5 minutes/wash) with PBS, incubated for 30 minutes at room temperature with Alexa Fluor 488 goat anti-rabbit and Alexa Fluor 568 goat anti-mouse antibodies in staining solution, and washed 3 times with PBS (5 minutes/wash). More highly magnified images of areas shown in the larger boxes are shown below the boxes. Confirmation that APLP2 and endocytosed folded HLA class I molecules are located together in vesicles in S2-013 cells was obtained by taking z-section images. Serial z-section images were obtained every $0.4 \mu \mathrm{m}$, and the crosshairs and arrows depict common membrane structures on a representative photomicrograph containing both APLP2 (green) and human MHC class I (red). These data verify that the indicated APLP2- and HLA-containing structures are identical (yellow) and not merely overlaid. Arrows indicate vesicles with co-localized endogenous APLP2 and endogenous, endocytosed HLA class I molecules. (C) As a negative control, S2-013 cells were incubated with mouse anti-transferrin receptor antibody at $4^{\circ} \mathrm{C}$, and then were warmed to $37^{\circ} \mathrm{C}$ for 15 minutes. The cells were treated with stripping solution $(0.5 \%$ acetic acid, $500 \mathrm{mM} \mathrm{NaCl}$ ) for 120 seconds to remove non-internalized surface-bound antibody, and fixed with $4 \%$ paraformaldehyde in PBS for 10 minutes. The cells were then incubated in staining solution with rabbit anti-APLP2 serum for 1 hour at room temperature, washed 3 times (5 minutes/wash) with PBS, incubated for 30 minutes at room temperature with Alexa Fluor 488 goat anti-rabbit and Alexa Fluor 568 goat anti-mouse antibodies in staining solution, and washed 3 times with PBS (5 minutes/wash). The insets depict more highly magnified images of areas shown in the larger boxes. Green = APLP2; red = transferrin receptor. For (A), (B), and (C), images were analyzed on a Zeiss LSM 5 Pascal confocal microscope and bar $=10 \mu \mathrm{m}$. 

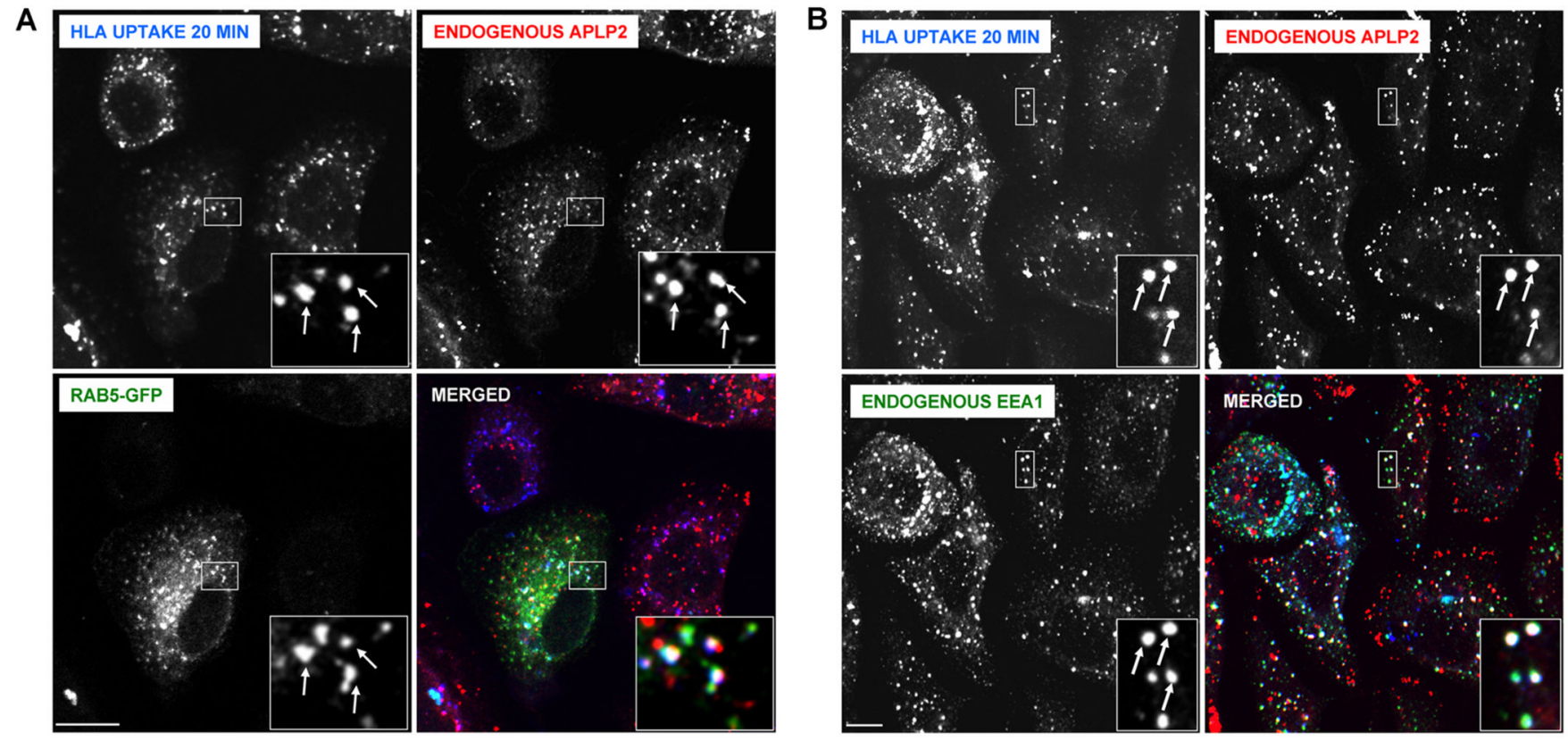

Figure 3.

In pancreatic tumor S2-013 cells, APLP2 molecules were demonstrated to be co-localized with folded HLA class I molecules in vesicular structures containing markers of early endosomes. (A) S2-013 cells were transfected with Rab5-GFP using Effectene reagent. At 24 hours posttransfection, the cells were incubated with mouse anti-HLA-A,B,C antibody $(\mathrm{W} 6 / 32)$ at $4^{\circ} \mathrm{C}$, then warmed to $37^{\circ} \mathrm{C}$ for 20 minutes. The cells were treated with stripping solution $(0.5 \%$ acetic acid, $500 \mathrm{mM} \mathrm{NaCl}$ ) for 120 seconds to remove non-internalized surface-bound antibody, and fixed with 4\% paraformaldehyde in PBS for 10 minutes. The cells were then incubated in staining solution with rabbit anti-APLP2 serum for 1 hour at room temperature, washed 3 times (5 minutes/wash) with PBS, incubated for 30 minutes at room temperature with Alexa Fluor 568 goat anti-rabbit and Alexa Fluor 405 goat anti-mouse antibodies in staining solution, and washed 3 times with PBS (5 minutes/wash). Blue $=$ HLA class I, red $=$ APLP2; green $=$ Rab5GFP; white $=$ merged. Arrows indicate some of the vesicles with co-localized HLA class I, APLP2, and Rab5-GFP. (B) S2-013 cells were incubated with mouse anti-HLA-A,B,C antibody (W6/32, which is an IgG2a antibody) at $4^{\circ} \mathrm{C}$, then warmed to $37^{\circ} \mathrm{C}$ for 20 minutes. The cells were treated with stripping solution $(0.5 \%$ acetic acid, $500 \mathrm{mM} \mathrm{NaCl})$ for 120 seconds to remove non-internalized antibody, and fixed with 4\% paraformaldehyde in PBS for 10 minutes. The cells were then incubated for 30 minutes in staining solution with Alexa Fluor 405 goat anti-mouse antibody. After 3 washes of 5 minutes each with PBS, the cells were incubated for 1 hour at room temperature in staining solution with rabbit anti-APLP2 serum and mouse anti-EEA1 antibody (IgG1). After an additional 3 washes in PBS (5 minutes/wash), the cells were incubated for 30 minutes at room temperature with Alexa Fluor 568 goat antirabbit antibody and Alexa Fluor 488 goat anti-mouse IgG1-specific antibody in staining solution, and washed 3 times with PBS (5 minutes/wash). Blue = HLA class I; red = APLP2; green $=$ EEA1; white $=$ merged. Arrows indicate some of the vesicles with co-localized HLA class I, APLP2, and EEA1. For both (A) and (B), images were analyzed on a Zeiss LSM 5 Pascal confocal microscope, $b a r=10 \mu \mathrm{m}$, and insets depict more highly magnified images of the areas shown in the larger boxes. 

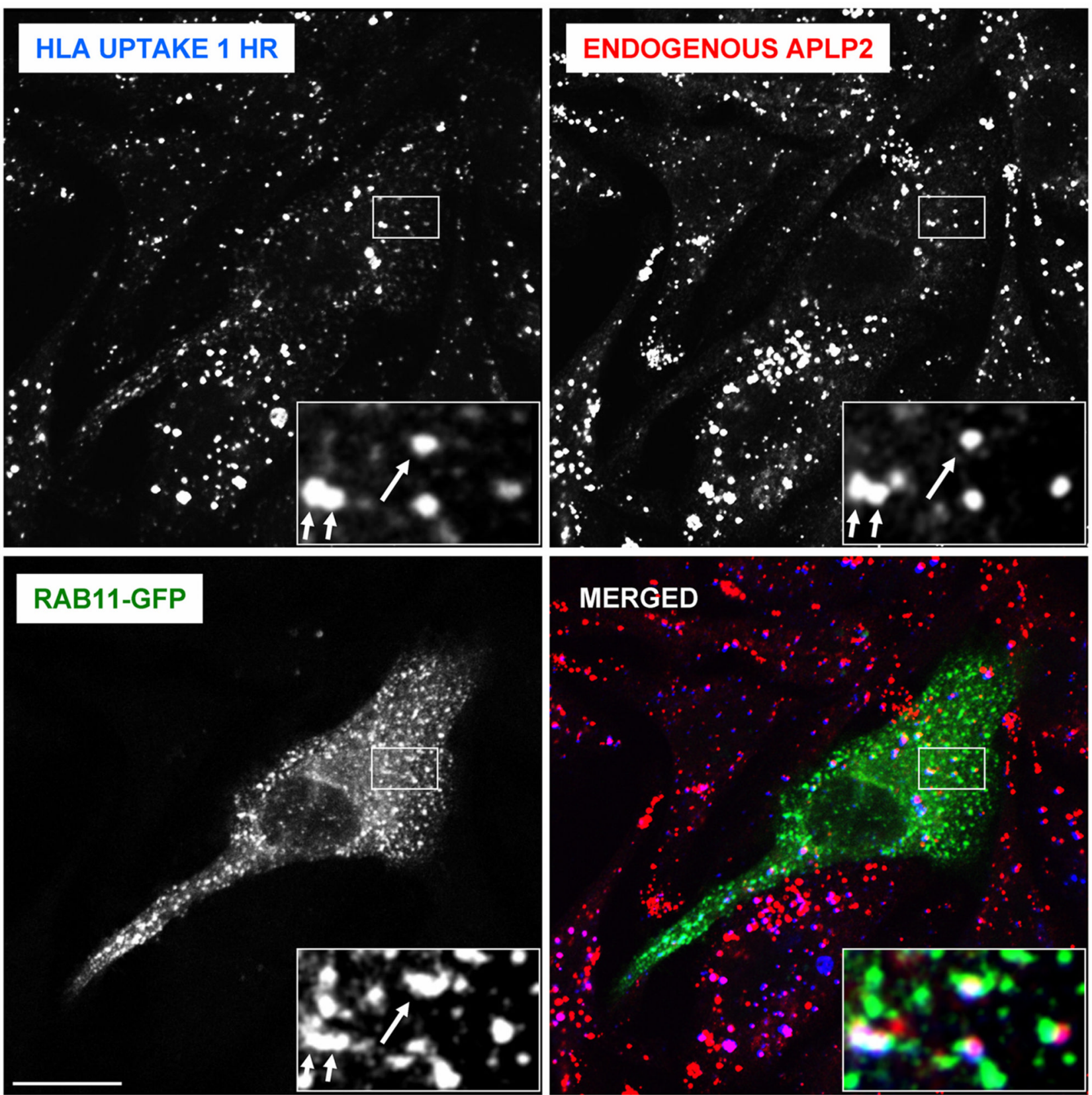

Figure 4.

APLP2 molecules were found to be co-localized with folded HLA class I molecules in vesicular structures containing a marker of recycling endosomes. S2-013 cells were transfected with Rab11-GFP using Effectene reagent. At 24 hours post-transfection, the cells were incubated with mouse anti-HLA-A,B,C antibody (W6/32) at $4^{\circ} \mathrm{C}$, then warmed to $37^{\circ} \mathrm{C}$ for 1 hour to allow sufficient time for the HLA class I molecules to reach the recycling endosomes. The cells were treated with stripping solution $(0.5 \%$ acetic acid, $500 \mathrm{mM} \mathrm{NaCl})$ for 120 seconds to remove non-internalized surface-bound antibody, and fixed with $4 \%$ paraformaldehyde in PBS for 10 minutes. The cells were then incubated in staining solution with rabbit anti-APLP2 serum for 1 hour at room temperature, washed 3 times ( 5 minutes/wash) with PBS, incubated for 30 minutes at room temperature with Alexa Fluor 568 goat anti-rabbit and Alexa Fluor 405 goat 
anti-mouse antibodies in staining solution, and washed 3 times with PBS (5 minutes/wash). Blue $=$ HLA class I; red $=$ APLP2 ; green $=$ Rab11-GFP. Arrows indicate some of the vesicles with co-localized HLA class I, APLP2, and Rab11-GFP. Images were analyzed on a Zeiss LSM 5 Pascal confocal microscope, bar $=10 \mu \mathrm{m}$, and insets depict more highly magnified images of the areas shown in the larger boxes. 
A
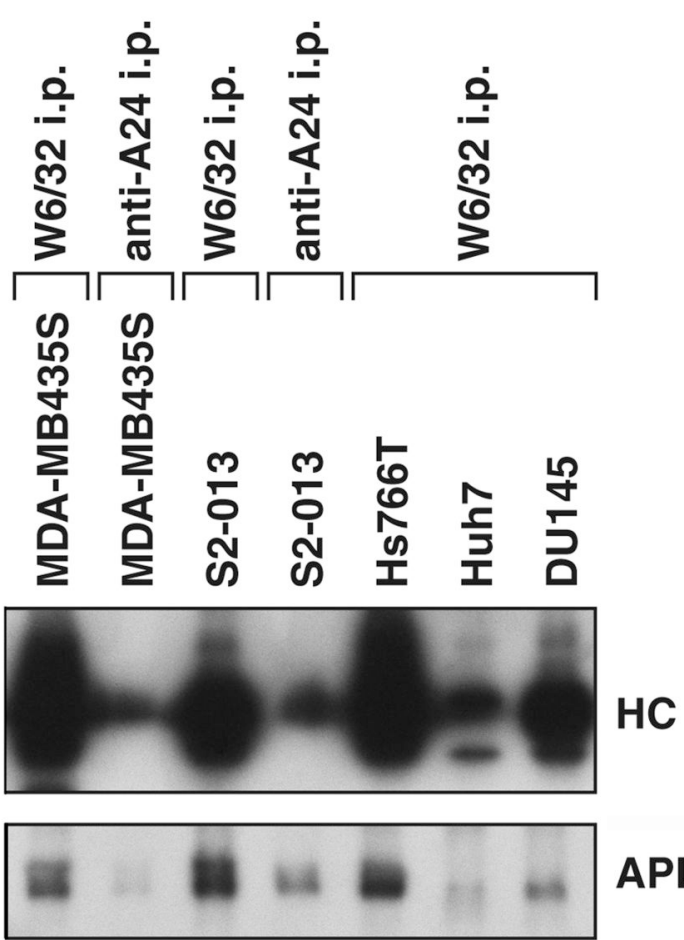

B

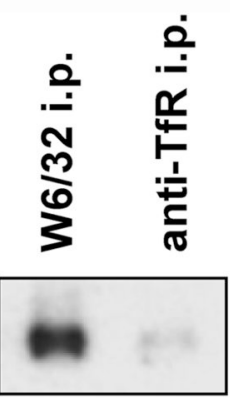

APLP2

C

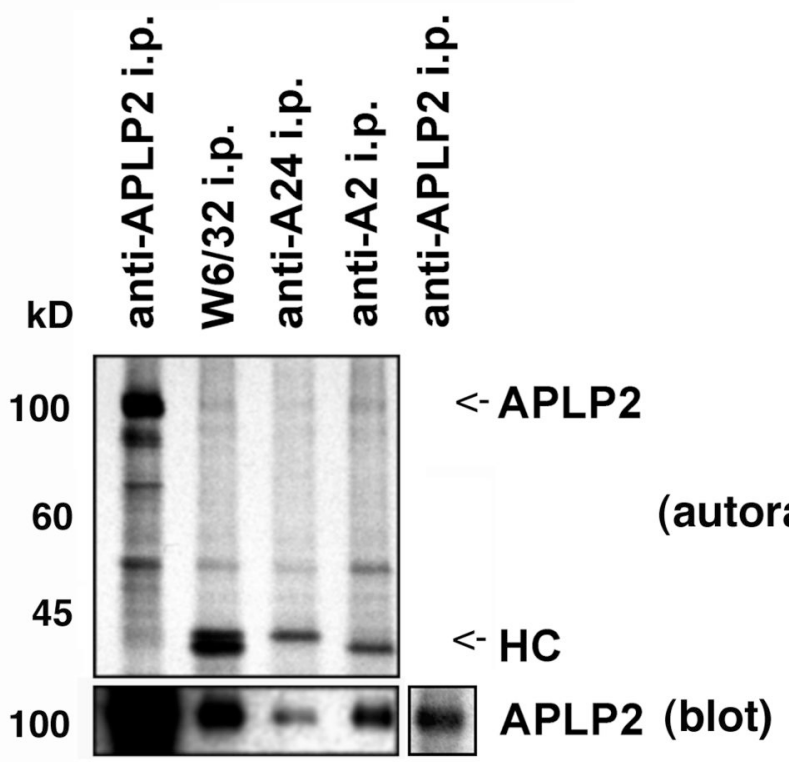

Figure 5.

APLP2 co-precipitated with HLA class I molecules in lysates of human tumor cell lines, more strongly with HLA-A2 than with HLA-A24. (A) Immunoprecipitations were performed on lysates of the indicated cell types with W6/32 or an antibody recognizing HLA-A24, and the immunoprecipitates were electrophoresed on $4 \rightarrow 20 \%$ acrylamide Tris-glycine gels, transferred to a Western blot, and probed with $\mathrm{HC} 10$ for the immunoprecipitated HLA class I heavy chains (HC), or with an antiserum recognizing APLP2. (B) HLA class I molecules and transferrin receptors (as a negative control) were immunoprecipitated from lysates of S2-013 cells with W6/32 and anti-transferrin receptor serum, respectively. The immunoprecipitates were electrophoresed on a $4 \rightarrow 20 \%$ acrylamide Tris-glycine gel, blotted, and probed with antiAPLP2 serum. (C) Immunoprecipitations were done on lysates of ${ }^{35} \mathrm{~S}$-methionine/cysteinelabeled pancreatic tumor S2-013 cells with an antiserum recognizing APLP2, the W6/32 antibody, an antibody recognizing HLA-A24, or an antibody recognizing HLA-A2. The samples were electrophoresed on $4 \rightarrow 20 \%$ acrylamide Tris-glycine gels. For autoradiography, the proteins were transferred from the gel to a membrane that was dried and exposed to Kodak BioMax film (upper panel, labeled as autorad), and also transferred to a second membrane that was probed with an antiserum recognizing APLP2 (bottom panel, labeled as blot). The APLP2 and heavy chain (HC) bands are indicated on the autoradiograph with arrows (the HLA-A24 and $-\mathrm{A} 2$ bands are of slightly different molecular weights). The additional section on the far 
right of the bottom panel shows a lighter exposure of the APLP2 blot so that the APLP2 band can be seen more clearly. 
A

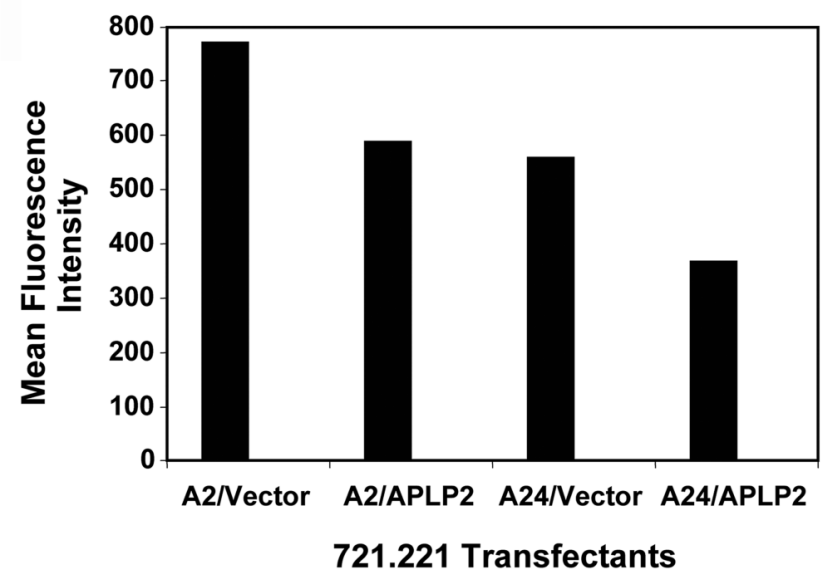

B

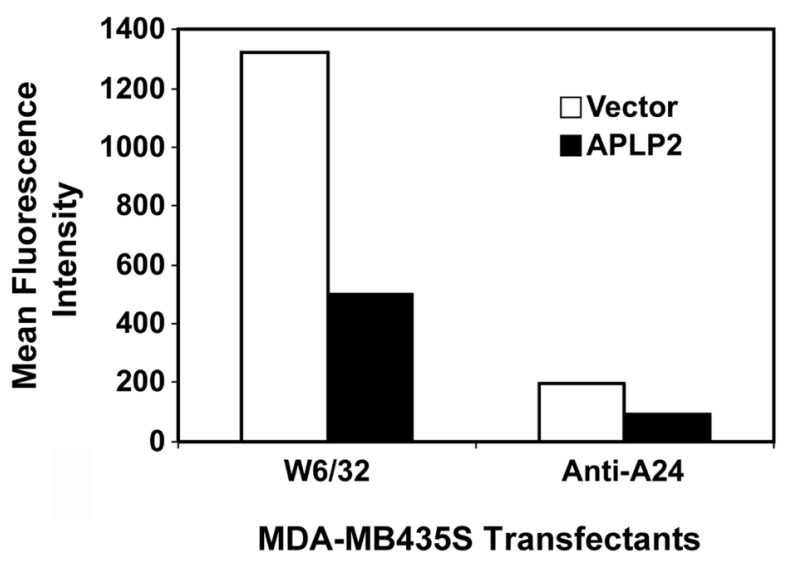

Figure 6.

(A) APLP2 transfectants of 721.221-A24 and 721.221-A2 cells had decreased cell surface HLA class I expression compared to vector-only transfectants. The bar graph displays the cell surface expression of HLA-A24 or HLA-A2 molecules at the surface of 721.221 cells transfected for 24 hours with vector only (pCMVTag4A) or with an APLP2 cDNA in pCMVTag4A. The cells were stained with either anti-A2 antibody (BB7.2) or anti-HLA-A24 antibody, washed and stained with phycoerythrin-labeled secondary antibody, and analyzed on a BD FACSCalibur. Separate results indicating that increased expression of APLP2 reduced HLA-A24 surface expression on transfected 721.221 cells were obtained in a separate experiment. (B) APLP2 transfectants of breast carcinoma MDA-MB435S cells had decreased cell surface HLA class I expression compared to vector-only transfectants. The bar graph displays the cell surface expression of total $\left(\mathrm{W6} / 32^{+}\right)$HLA class I molecules or HLA-A24 molecules at the surface of MDA-MB435S cells stably transfected with vector only (pCMVTag4A) or with an APLP2 cDNA in pCMVTag4A. The cells were stained with either W6/32 or with anti-HLA-A24 antibody, washed and stained with phycoerythrin-labeled secondary antibody, and analyzed on a BD FACSCalibur. Separate results indicating that elevated expression of APLP2 reduced HLA-A24 surface expression on APLP2-transfected MDA-MB435S cells were also obtained in two additional experiments. 
A
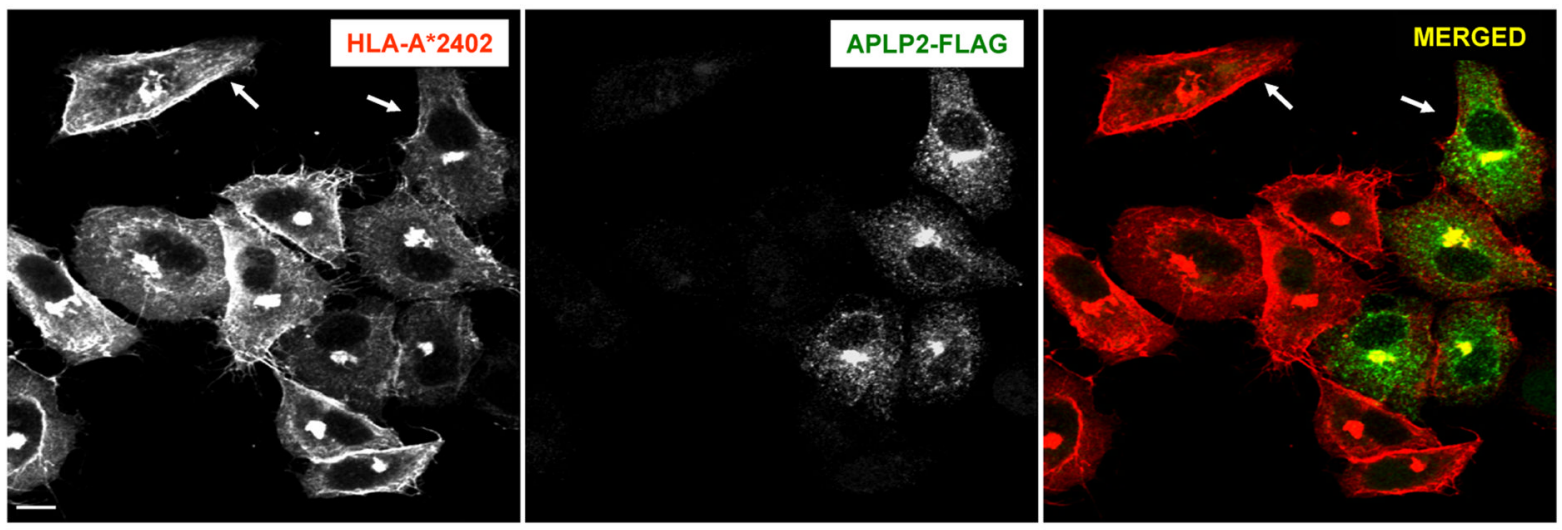

B
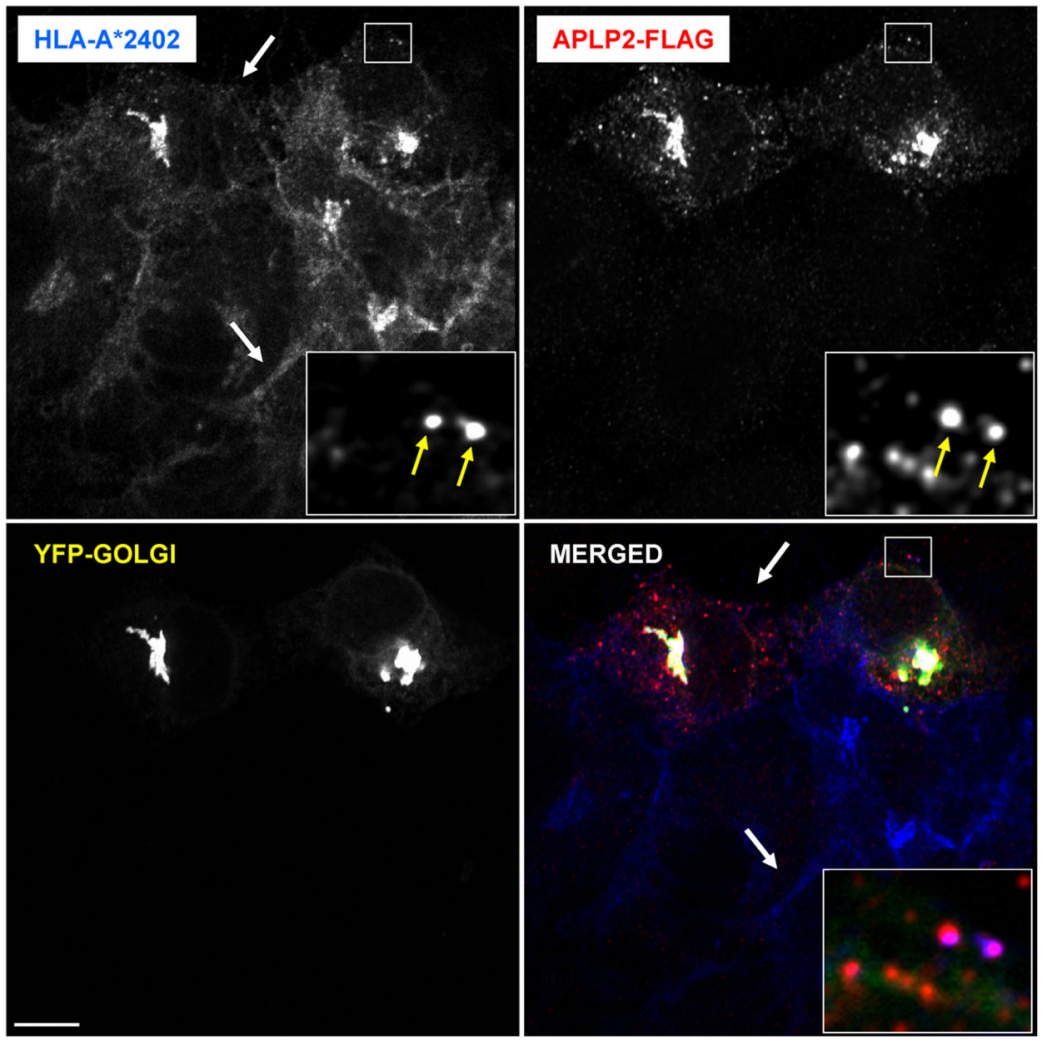

Figure 7.

Elevated expression of APLP2 in HeLa cells reduced the amount of HLA-A24 at the cell surface. (A) HeLa cells stably expressing HLA-A*2402 were transfected with APLP2-FLAG by the use of Effectene. At 24 hours, the cells were fixed by treatment with $4 \%$

paraformaldehyde in PBS for 10 minutes, incubated with primary antibodies (mouse anti-HLAA24 and rabbit anti-FLAG antibodies) in staining solution for 1 hour at room temperature, and washed (3 times in PBS, 5 minutes/wash). The cells were then incubated with fluorochromeconjugated secondary antibodies (Alexa Fluor 568 goat anti-mouse antibody and Alexa Fluor 488 goat anti-rabbit antibody) in staining solution for 30 minutes at room temperature, and washed again 3 times with PBS for 5 minutes/wash. The arrows at the upper left of the first and third panels point to an area of bright staining of HLA-A $* 2402$ molecules at the surface of HeLa-A24 cells, and the arrows at the upper right of the same panels point to an area of relatively weak staining on a HeLa-A24 cell transfected with APLP2-FLAG. (B) HeLa cells 
stably expressing HLA-A*2402 were transfected with APLP2-FLAG and YFP-Golgi by the use of Effectene. At 24 hours, the cells were fixed by treatment with $4 \%$ paraformaldehyde in PBS for 10 minutes, incubated with primary antibodies (mouse anti-HLA-A24 and rabbit antiFLAG antibodies) in staining solution for 1 hour at room temperature, and washed (3 times in PBS, 5 minutes/wash). The cells were then incubated with fluorochrome-conjugated secondary antibodies (Alexa Fluor 405 goat anti-mouse antibody and Alexa Fluor 568 goat anti-rabbit antibody) in staining solution for 30 minutes at room temperature, and washed again 3 times with PBS for 5 minutes/wash. Arrows in the larger boxes indicate less HLA-A*2402 at the plasma membrane of a cell transfected with APLP2-FLAG (top arrow) compared to more HLA-A*2402 at the surface of a cell that was not transiently transfected with APLP2-FLAG. Insets depict more highly magnified images of the areas shown in the larger boxes. The arrows within the insets indicate peripheral vesicles in which HLA-A*2402 (blue) and APLP2-FLAG (red) are co-localized (magenta). Blue = HLA class I; red = APLP2-FLAG; yellow = YFPGolgi; white $=$ merged. For both $(\mathrm{A})$ and $(\mathrm{B})$ images were analyzed on a Zeiss LSM 5 Pascal confocal microscope and bar $=10 \mu \mathrm{m}$. 


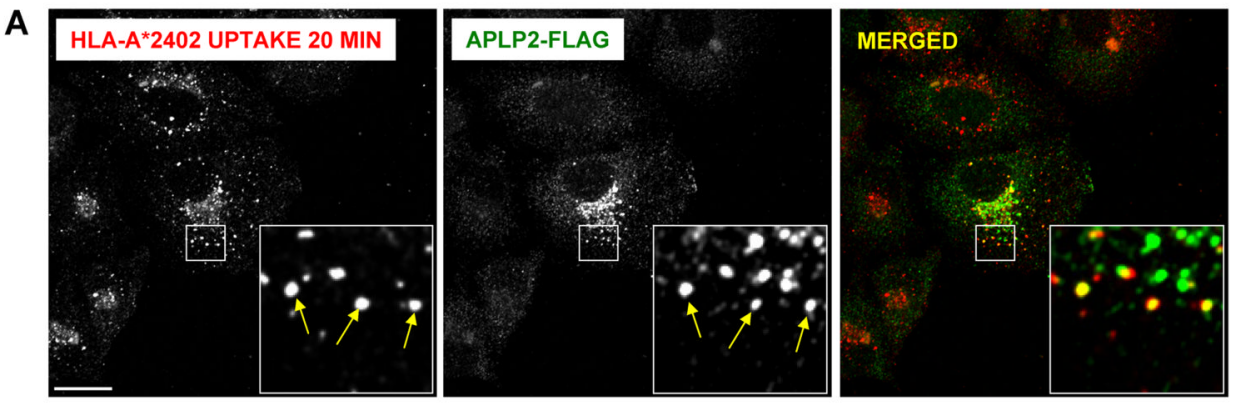

B
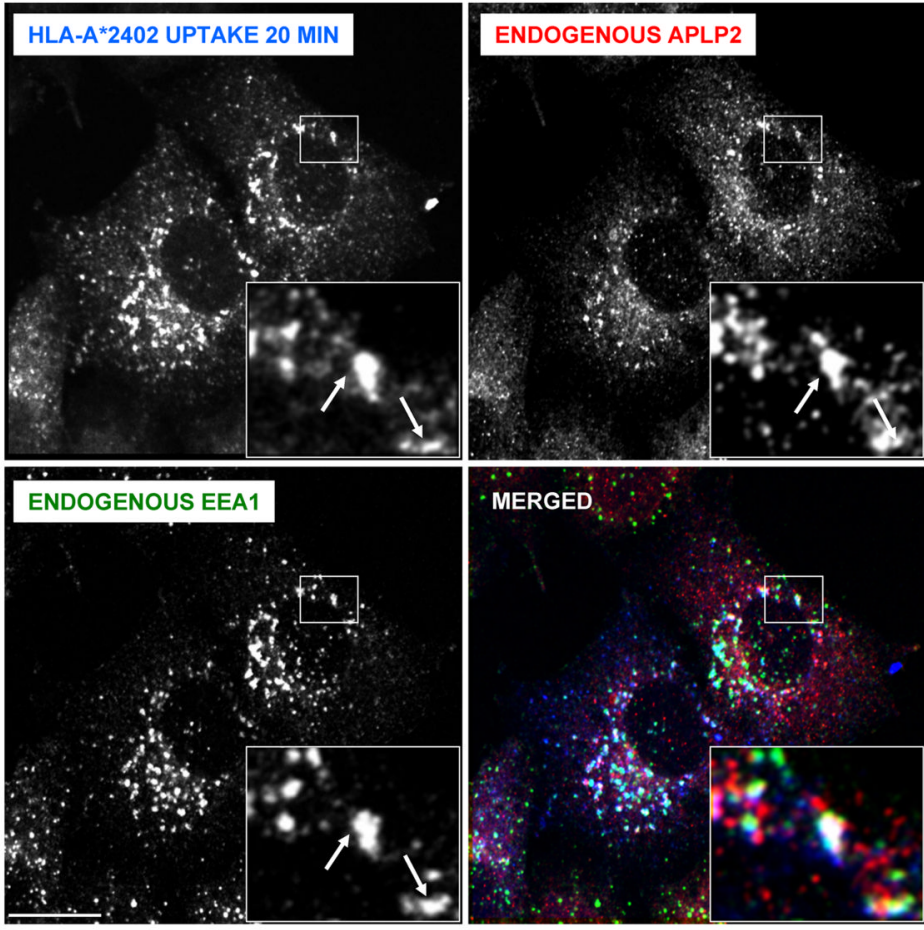

Figure 8.

Endocytosed HLA-A*2402 molecules co-localized with APLP2 in early endosomes. (A) HeLa cells stably expressing HLA-A*2402 were transfected with APLP2-FLAG by the use of the Effectene transfection reagent. At 24 hours, the cells were pulsed with anti-HLA-A24 antibody for 20 minutes. At the end of the pulse period, remaining cell surface-bound antibodies were removed by incubating the cells with stripping solution $(0.5 \%$ acetic acid, $500 \mathrm{mM} \mathrm{NaCl})$ for 90 seconds, and the cells were fixed by incubation with 4\% paraformaldehyde in PBS for 10 minutes. The cells were then incubated in staining solution with rabbit anti-APLP2 serum for 1 hour at room temperature, washed 3 times ( 5 minutes/wash) with PBS, incubated for 30 minutes at room temperature with Alexa Fluor 568 goat anti-mouse and Alexa Fluor 488 goat anti-rabbit antibodies in staining solution, and washed 3 times with PBS (5 minutes/wash). Red $=$ HLA-A $* 2402$; green $=$ APLP2-FLAG; yellow $=$ merged. Insets represent more highly magnified images of the areas that are in the larger boxes. Arrows within the insets indicate some of the vesicles in which HLA-A*2402 and APLP2-FLAG are co-localized. (B) HeLa cells stably expressing HLA-A $* 2402$ were pulsed with anti-HLA-A24 antibody for 20 minutes. After the pulse period, remaining surface-bound antibodies were removed by treating the cells with stripping solution $(0.5 \%$ acetic acid, $500 \mathrm{mM} \mathrm{NaCl}$ ) for 90 seconds, and the cells were fixed by incubation in $4 \%$ paraformaldehyde in PBS for 10 minutes. The cells were then incubated in staining solution with Alexa Fluor goat anti-mouse 405 antibody for $30 \mathrm{~min}$. After 
3 washes of 5 minutes each with PBS, the cells were stained with rabbit anti-APLP2 serum and anti-EEA1 (IgG1) antibody for 1 hour at room temperature, washed 3 times (5 minutes/ wash) with PBS, incubated for 30 minutes at room temperature with Alexa Fluor 568 goat antirabbit and Alexa Fluor 488 goat anti-mouse IgG1 antibodies in staining solution, and washed 3 times with PBS (5 minutes/wash). Blue $=$ HLA-A $* 2402$; red $=$ APLP2; green $=$ EEA1; white $=$ merged. Insets represent more highly magnified images of the areas that are in the larger boxes. Arrows within the insets indicate some of the vesicles in which HLA-A*2402, APLP2, and EEA1 are co-localized. For both (A) and (B), the images were analyzed on a Zeiss LSM 5 Pascal confocal microscope and $\mathrm{bar}=10 \mu \mathrm{m}$. 